\title{
The Pricing of Non-Independent Risks Under Constant Absolute Risk Aversion
}

\author{
by Emilio C. Venezian and Dongsae Cho*
}

\section{Introduction}

This paper analyses the conditions under which an insurer will undertake to write policies for risks that are interdependent. The two areas will be of special interest:

1. the relation between the number of risks assumed and the premium that will be charged, and

2. the effect of interdependence on this relation.

In essence we are trying to establish the insurance company's supply function for coverage of interdependent risks. Similar considerations are used to describe the demand function. The existence of a competitive market equilibrium, even under conditions of perfect knowledge, has not been established.

Most of the analyses dealing with the setting of premium have dealt with risks which are independent from each other. Financial models based on the capital asset pricing model, such as those of Fairley [3], Hill [5], and Urrutia [8], or on arbitrage pricing theory, such as those of Kraus and Ross [7] and Urrutia [9] are sensitive to interdependence only to the extent that such interdependence is correlated with the performance of the economy as a whole or with the performance of specific segments of the economy. From a broader economic context, however, the interdependence of risks may have a substantial influence on the decision of whether to assume the specific risks or how many risks of a given type (or in a given area) should be underwritten.

This paper explores interdependence from the perspective of an insurer that is assumed to be averse to risk. Much of the economic and financial literature is founded on the assumption that firms, and particularly insurance companies, are risk neutral and reach decisions on the basis of the expected value of the profit of the possible outcomes. Such a perspective may be appropriate in the context of independent risks, a context in which the profits associated with possible outcomes for a large portfolio of risks are in a relatively small neighborhood

* Emilio Venezian is Associate Professor in the Department of Business Administration at Rutgers University. Don Cho is Assistant Professor in the School of Management at the University of Minnesota. An earlier version of this paper was presented in September 1985 in Brussels at the 12th Seminar of the European Group of Risk and Insurance Economists. 
around the expected value of that profit. With positively correlated risks, however, the probability distribution of the outcomes may be much more dispersed and much less symmetrical. The probability of insolvency will generally be much larger. In this context it appears reasonable to as sume that insurers are averse to risk. Indeed, if insurers were not averse to risk, regulatory authorities charged with the safeguarding of the solvency of insurers might intervene and force a price that reflects the riskiness of the coverage under examination. We will assume that the insurer's risk aversion can be characterized by constant absolute risk aversion. This form of risk aversion, leading to an exponential utility function, has the virtue that as the risk aversion coefficient becomes closer to zero the utility curve approachs linearity; in the limit the results are applicable to the case of risk neutrality. The function also is convenient from the analytical point of view since it leads to explicit results. ${ }^{1}$

The model developed in this paper will deal with a form of interdependence which leads to positive correlation among the losses. Other forms of interdependence could be postulated and forms that lead to negative correlation might also be of interest. Positive correlation appears the more important because it will increase the probability of insolvency and provides an example of a situation in which the usual assumption of risk neutrality and of no costs associated with insolvency may provide a poor approximation.

It is also worth noting that the model presented here is one in which all risks are identical, except the possibility that the risk aversion coefficient varies among the economic agents. The model also assumes that the losses are neither predictable nor preventable, so there are no issues of self-selection or moral hazard.

It would be interesting to determine the effect of reinsurance on the supply of insurance. The existing literature on reinsurance, along with that of primary insurance, however, has not devoted much attention to pricing in the presence of interdependent losses. The major results regarding diversification through reinsurance and optimal reinsurance contracts all depend on the assumption that losses are independent and are, accordingly, not applicable to the situation being considered here. The analysis of the effect of reinsurance is outside the scope of this paper.

\section{Relation between premiums and demand}

Consider a situation in which a potential purchaser of insurance faces a probability $p_{1}$ of losses from "non-catastrophic" events whose occurrences are independent of each other, and a probability $p_{2}$ of losses from "catastrophic" events which, when they occur, affect all units within a defined group such as a geographic area. ${ }^{2}$ As a guide to visualising the model one might think in terms of insurance of physical structures, such as houses. These are subject

${ }^{1}$ Utility functions with constant proportional risk aversion, the logarithmic and power functions, have been advocated by Copeland and Weston [1], Friend and Blume [4], Kenney and Raifa [6] among others. These functions approach minus infinity as wealth approaches zero and yield complex numbers for negative wealth. They are not useful when there is a non-zero probability of insolvency.

${ }^{2}$ Although the model is equally valid when non-independence of losses is caused by characteristics other than geography, such as exposure to different hazardous materials or different legal systems, this paper will use the geographic example throughout. 
to loss through events which are essentially independent, such as through fire. They are also subject to losses which tend to affect all structures in a geographical area in the same way, such as floods earthquakes, hurricanes, and other large-scale phenomena.

Table 1: Loss prospects

\begin{tabular}{cccc}
\hline \multicolumn{2}{c}{ Event Combination } & Probability & $\begin{array}{c}\text { Contingent } \\
\text { Loss }\end{array}$ \\
Independent & Catastrophe & $\left(1-p_{1}\right)\left(1-p_{2}\right)$ & $L_{0}^{*}$ \\
Yes & No & $p_{1}\left(1-p_{2}\right)$ & $L_{1}$ \\
No & No & $p_{2}\left(1-p_{1}\right)$ & $L_{2}$ \\
Yes & Yes & $p_{1} p_{2}$ & $L_{3}$ \\
\hline
\end{tabular}

* Note that the loss in this case is zero; the use of a subscripted variable does, however, simplify some of the equations.

The loss prospects for a given potential purchaser are displayed in Table 1. Using the symbols defined in this table, the actuarial value of the loss is $p_{1} L_{1}+p_{2} L_{2}+p_{1} p_{2}\left(L_{3}-L_{1}-L_{2}\right)$. If $L_{3}$, the loss when both independent and catastrophic events occur, were the maximum of those under the single contingencies; that is, $L_{3}$ is the maximum of $L_{1}$ and $L_{2}$, the actuarial value of the loss would be $p_{1} L_{1}+p_{2} L_{2}-p_{1} p_{2} \min \left(L_{1}, L_{2}\right)$.

A potential purchaser with constant absolute risk aversion coefficient $r_{A}$ and initial wealth $W_{A}$ has a utility function:

$$
U\left(W_{A}\right)=\frac{1-e^{-r_{A} W_{A}}}{r_{A}}
$$

If insolvency of the insurer does not play a role in the individual's decision processes, then the individual will purchase full insurance against the contingent losses provided that it is available at a premium no greater than $P_{A}$ :

$$
p_{A}=\frac{1}{r_{A}} \ln \left(1+p_{1} Z_{1}+p_{2} Z_{2}+p_{1} p_{2}\left(Z_{3}-Z_{1}-Z_{2}\right)\right)
$$

where $Z_{i}=e^{r_{A} L_{i}}-1$ 
Taken together with the distribution of risk aversion coefficients in the population, this equation establishes the relation between premium and demand for insurance.

\section{Relation between premiums and supply}

As a counterpart, the utility functions of the insurers, taken jointly, will establish the relation between premium and supply of insurance. This relation can be developed by determining whether the insurer's expected utility given that it assumes a number of risks at a given price is higher than its utility if it assumes no insurance. The derivation of the relation for the potential supplier of insurance is somewhat more complicated than that relating to the potential purchaser of insurance because the interdependence of event combinations must be taken into account. The framework presented here can be used to handle quite complex situations, but the results may not be expressible in simple analytic terms.

This paper examines the case in which the insurer writes the same number of policies, $N_{R}$, in each of $K$ areas, each of which has a probability $p_{2}$ of suffering a catastrophic loss, this loss being independent of the occurrence of catastrophies in other areas. The probabilities of all event combinations can then be expressed readily if they are made conditional on $k$, the number of areas affected by catastrophic events.

The number of units which are not affected by a catastrophic event is $(K-k) N_{R}$. The conditional probability that exactly $n_{1}$ units which were not affected by a catastrophic event will suffer a loss due to a non-catastrophic event is: ${ }^{3}$

$$
p\left(n_{1} / k\right)=\frac{\left((K-k) N_{R}\right) !}{n_{1} !\left((K-k) N_{R}-n_{1}\right) !} p_{1}^{n_{1}}\left(1-p_{1}\right)(K-k) N_{R}-n_{1} .
$$

The number of units which are affected by a catastrophic event is $k N_{R}$. The conditional probability that exactly $n_{3}$ of these will suffer a loss due to a non-catastrophic event is: ${ }^{4}$

${ }^{3}$ If instead of being binomially-distributed the number of independent losses were Poisson-distributed with expected frequency $p_{1}$ per unit, then the number of losses would be:

$$
p\left(n_{1} / k\right)=\frac{\left[p_{1} N_{R}(K-k)\right]^{n_{1}}}{n_{1} !} e^{-p_{1}(K-k) N_{R}} .
$$

The range of $n_{1}$ would be all positive integers.

${ }^{4}$ With a Poisson distribution for independent losses this expression would take the form:

$$
p\left(n_{3} / k\right)=\frac{\left(p_{1} k N_{R}\right)^{n_{3}}}{n_{3} !} e^{-p_{1} k N_{R}} .
$$

The range of $n_{3}$ would be all positive integers. 


$$
p\left(n_{3} / k\right)=\frac{\left(k N_{R}\right) !}{n_{3} !\left(k N_{R}-n_{3}\right) !} p_{1}^{n_{3}}\left(1-p_{1}\right)^{k N_{R}-n_{3}} .
$$

The number of units affected by a catastrophic event which do not suffer a non-catastrophic loss is $k N_{R}-n_{3}$.

The probability that exactly $k$ areas are affected by catastrophic events is simply:

$$
p(k)=\frac{K !}{k !(K-k) !} p_{2}^{k}\left(1-p_{2}\right)^{K-k} .
$$

Since $n_{1}$ and $n_{3}$ are independent, on the condition that the number of affected areas is $k$, the probability of the combined event can then be written as:

$$
p\left(n_{1}, n_{3}, k\right)=p(k) p\left(n_{1}, n_{3} / k\right)=p(k) p\left(n_{1} / k\right) p\left(n_{3} / k\right) .
$$

Hence the expected utility, $E(U)$, of an insurer with initial wealth $W_{B}$ which writes $N_{R}$ policies in each of $K$ areas at a premium per policy of $P_{B}$ is given by:

$$
E(U)=\sum_{k=0}^{K} \sum_{n_{1}=0}^{(K-k) N_{R}} \sum_{n_{3}=0}^{k N_{R}} p\left(n_{1}, n_{3}, k\right) U\left(W\left(n_{1}, n_{3}, k\right)\right),
$$

where $W\left(n_{1}, n_{3}, k\right)=W_{B}+K N_{R} P_{B}-n_{1} L_{1}-\left(k N_{R}-n_{3}\right) L_{2}-n_{3} L_{3}$ is the wealth of the insurer for the realization $n_{1}, n_{3}, k$. After some algebra this reduces to: ${ }^{5}$

$$
\left.E(U)=\frac{1}{r_{B}}\left[1-e^{-r_{B}\left(W_{B}+K N_{R} P_{B}\right)}\left(\left(1-p_{2}\right) Q_{0} N_{R}+p_{2} Q_{2} N_{R}\right)\right)\right],
$$

where $r_{B}$ is the risk aversion coefficient of the insurance company and

$$
Q_{i}=\left(1-p_{1}\right) e^{r_{B} L_{i}}+p_{1} e^{r_{B} L_{i+1}} .
$$

The insurer will assume those risks only if the expected value of its utility after their assumption is at least as large as its utility at the initial wealth $W_{B}$. This condition imposes a restriction on the premium and, if the market is reasonably competitive the restriction becomes the equation:

${ }^{5}$ If the number of independent losses were Poisson-distributed, the summations over $n_{1}$ and $n_{3}$ would be over all positive integers. The corresponding relation is identical to Equation 8 but with:

$$
Q_{i}=e^{r_{B} L_{i}+p_{1}\left[e^{r_{B}\left(L_{i+1}-L_{i}\right)}-1\right]}
$$

Subsequent equations still hold provided the appropriate values of $Q_{i}$ are used. 


$$
P_{B}=\frac{K}{r_{B} N_{T}} \ln \left[\left(1-p_{2}\right) Q_{0}^{\left(N_{T} / K\right)}+p_{2} Q^{\left(N_{T} / K\right)}\right] \text {, }
$$

where $N_{T}=K N_{R}$

This expression defines the relationship between premium and supply for the individual insurance company. Taken together with the distribution of the aversion coefficient among insurers and with the dynamics of competition, this expression would define the premiumsupply relation for the insurance market.

\section{Characteristics of the supply function of the firm}

Equation (9) is, apart from a constant, of the form:

$$
f(x)=\frac{1}{x} \ln \left(\left(1-a^{\prime}\right) b_{1}^{x}+a^{\prime} b_{2}^{x}\right)
$$

As shown in the Appendix, this represents a monotonic relation with premium $f(x)$ increasing as $x$ increases. Since $x$ is identified with $N_{R}=N_{T} / K$, it follows that if the total number of policies per area is to be increased, a higher premium must be charged. In this respect, Equation (9) exhibits the classic characteristics of a supply function for the firm. On the other hand if the number of areas, $K$, is increased while holding the total number of policies, $N_{T}$, constant, $x$ is decreased and this would be accompanied by a decrease in the required premium. Proportional increases in $K$ and $N_{T}$, so that $N_{R}$ remains constant, do not affect the required premium. Increases in scale of operation by increasing geographic diversification while decreasing the number of policies in individual areas are more beneficial than increases in scale at constant or increasing geographic concentration.

As shown in the Appendix, as the number of risks per area increases without bound the required premium approaches asymptotically a value $P_{B}(\infty)$ which is the maximum of $\ln Q_{0}$ and $\ln Q_{1}$. As a rule, it could be expected that the occurrence of a catastrophic event would not reduce the severity of losses from non-catastrophic events that affect the same insured unit, so that $L_{3}>L_{1}$. Similarly, units that have not suffered from non-catastrophic events would have a higher severity if a catastrophe occurred than if it did not, so that $L_{2}>L_{0}$. In that case, $Q_{2}$ is greater than $Q_{0}$, and a lower bound on $Q_{2}$ can be obtained by assuming that $L_{3}=L_{2}$. The corresponding lower bound on $P_{B}(\infty)$ is $L_{2}$, the value of a non-catastrophic loss. ${ }^{6}$

At that price insurance demand would be limited to conditions of very high risk aversion and probabilitities of loss close to unity. The price can be kept lower, and the demand higher, by limiting the exposure of the insurance company. This characteristics of the market suggests that when catastrophic losses are possible it is important for insurers to recognize the

${ }^{6}$ When the independent and catastrophic losses do not both have binomial distributions, the lower bound will have a different functional form. 
potential consequences of non-independent losses, limit their exposure to such losses, and diversify. By these strategies premiums can be kept at a level which will be attractive to a large segment of potential purchasers.

\section{Structure of the premium}

Since $N_{R}$ is a discrete variable whereas $x$ is a continuous one, the limit of $P_{B}$ as $x$ goes to zero represents the premium that would be charged by an insurer writing an infinitessimal share of a single policy. The symbol $P_{B}(e)$ will be used to denote this limit. From Equation(9) and the relations shown in the Appendix, this value is found to be:

$$
P_{B}(e)=\frac{1}{r_{B}}\left[\left(1-p_{2}\right) \ln \left(Q_{0}\right)+p_{2} \ln \left(Q_{2}\right)\right] .
$$

As $r_{B}$ approaches zero, the quantities $\ln \left(Q_{i}\right) / r_{B}$ take on the indeterminate form zero over zero. Applying l'Hopital's rule:

$$
\lim _{r_{B} \rightarrow 0} \frac{\ln \left(Q_{i}\right)}{r_{B}}=\lim _{r_{B} \rightarrow 0} \frac{d\left(Q_{i}\right) / d r_{B}}{Q_{i}}=\left(1-p_{1}\right) L_{i}+p_{1} L_{i+1} .
$$

Using this equation, the limit of $P_{B}(e)$ as $r_{B}$ approaches zero is found to be:

$$
\begin{aligned}
P_{B}(0)=\lim _{r_{B} \rightarrow 0} P_{B}(e)= & \left(1-p_{1}\right)\left(1-p_{2}\right) L_{0}+p_{1}\left(1-p_{2}\right) L_{1} \\
& +p_{2}\left(1-p_{1}\right) L_{2}+p_{1} p_{2} L_{3} .
\end{aligned}
$$

In simple words, this represents the actuarial value of the losses. The difference $P_{B}(e)-$ $P_{B}(0)$ is a function of the risk aversion coefficient, $r_{B}$, the loss probabilities, $p_{1}$ and $p_{2}$, and the loss severities, $L_{i}$. By definition, it vanishes when the risk aversion coefficient approaches zero. Moreover, it is a positive and increasing function of the risk aversion coefficient as can be demonstrated by noting that, apart from positive constant it is the sum of functions of the form of Equation (10), with $x=r_{B}$. It does not vanish when the probability of a catastrophic event vanishes. It is appropriate, therefore, to characterize it as a component of premium which is needed to compensate for risk aversion.

The difference between $P_{B}\left(N_{R}\right)$ and $P_{B}(e)$ represents a third component of the premium. This difference is positive and is a monotonic function of $N_{R}$, since $P_{B}\left(N_{R}\right)$ was proved to be monotonic increasing with $P_{B}(e)$ as its lower limit. This difference is also a function of the parameters $r_{B}, p_{i}$, and $L_{i}$. The fact that this component vanishes when the decision maker is risk neutral is of interest but does not help to characterize this component. Of greater interest is the fact that it vanishes identically when $p_{2}$ is zero and also in the limit as $N_{R}$ approaches zero. Although the last component vanishes whenever the probability of catastrophic events is zero, it does depend on the probability of independent events and on the number of policies assumed, so it cannot be characterized as an allowance for contingencies. It can, however, be characterized as a component of premium which is needed to allow for catastrophies and for capacity. 


\section{Are losses separable?}

The structure of the supply prices suggests that it may be worthwhile to consider insurance policies that cover the independent losses as contracts to be sold separately from those covering the "catastrophic" losses. There is, in fact, ample evidence that this phenomenon is seen in the market, at least in the United States. Some examples are:

1. earthquake and floor exclusions

2. war and nuclear damage exclusions

3. riot and civil disturbance exclusions.

Less visible, but along the same lines, are restrictions on the coverage of key corporate officers to no more than ten deaths occurring from personnel travelling in the same airplane.

Contractual exclusions and limitations, however, do not always succeed in separating the risks. For example, fire damage to a building must still be indemnified even if the fire resulted from a gas main ruptured by an earthquake. In other cases, there appears to be no practical way of framing the contract to achieve a separation. An example would be the exclusion of death during an epidemic from indemnification under a life insurance policy. The question of how "an epidemic" might be defined is compounded by the problem of defining just which causes of death are to be affected, the criteria that are to be used in establishing the cause of death, and the exact time and space relations that must apply.

The model developed in this paper suggests that attempts to set exclusions and restrictions such as those mentioned above may result from a need to keep insurance for the independent losses at an affordable level. When perfect separation of the risks can be achieved it is of interest to determine whether separation is in the best interests of the parties. A separation theorem would be attractive because of its analogy to capital asset valuation theory. The model presented here provides a way of examining this question.

Consider a potential buyer of insurance whose risk aversion coefficient is $r_{A}$ and a potential seller of insurance whose risk aversion coefficient is $r_{B}$. If the seller sells $N_{T}$ policies evenly allocated among $K$ areas, the price of full coverage, $P_{B}$, will be that given by Equation (9). If the only losses to the insurer are those from independent causes, then the price will be $P_{B}{ }^{*}$, and can be obtained from Equation (9) by setting $p_{2}$ to zero:

$$
P_{B}^{*}=\frac{1}{r_{B}} \ln \left[1+p_{1}\left(e^{r_{B} L_{1}}-1\right)\right] .
$$

If the consumer purchases coverage for both independent and catastrophic risks he will have an expected utility:

$$
E_{\mathrm{i}}(U)=\frac{1}{r_{A}}\left[1-e^{-r_{A}\left(W_{A}-P_{B}\right)}\right] .
$$

On the other hand, if the consumer purchases coverage for only the independent losses and decides to be self-insured for the catastrophic losses, his expected utility will be: 


$$
E_{2}(U)=\frac{\left(1-p_{2}\right)}{r_{A}}\left[1-e^{-r_{A}\left(W_{A}-P_{B}^{*}\right)}\right]
$$

(16)

$$
+\frac{p_{2}}{r_{A}}\left[1-e^{-r_{A}\left(W_{A}-P_{B}^{*}-L_{2}\right)}\right] \text {. }
$$

The total coverage will be preferable whenever:

$$
e^{r_{A} P_{B}}<e^{r_{A} P_{B} *}\left[1+p_{2}\left(e^{r_{A} L_{2}}-1\right)\right] .
$$

Taking logarithms and using the expressions for $P_{B}$ and $P_{B}{ }^{*}$, this inequality may be expressed as:

$$
\ln \left[1+p_{2}\left(e^{r_{A} L_{2}}-1\right)\right]>\frac{r_{A}}{r_{B} N_{R}} \ln \left[1-p_{2}+p_{2}\left[\frac{Q_{2}}{Q_{0}}\right]^{N_{R}}\right] .
$$

Since $Q_{2}>Q_{0}$, the theorems in the Appendix guarantee that the inequality will be satisfied whenever:

$$
1+p_{2}\left[e^{r_{A} L_{2}}-1\right]>\left[\frac{Q_{2}}{Q_{0}}\right]\left(r_{A} / r_{B}\right) .
$$

This expression may also be written as

(20)

$$
p_{2}>\frac{\left[\frac{\left(1-p_{1}\right) e^{r_{B} L_{2}}+p_{1} e^{r_{B} L_{3}}}{\left(1-p_{1}\right) e^{r_{B} L_{0}}+p_{1} e^{r_{1} L_{1}}}\right]^{\left(\mathrm{r}_{A} / r_{B}\right)}-1}{e^{r_{A} L_{2}}-1} .
$$

For sufficiently large values of $r_{A}$ the right hand side of this expression approaches the value

$$
\left[\frac{\left(1-p_{1}\right)+p_{1} \mathrm{e}^{r_{B}\left(L_{3}-L_{2}\right)}}{\left(1-p_{1}\right)+p_{1} e^{r_{B}\left(L_{1}-L_{0}\right)}}\right]
$$

Thus it is clear that for sufficiently large values of $r_{A}$ and sufficiently small values of $L_{3}-L_{2}$ there will be values of $p_{2}$ such that the purchaser will prefer to pay for total coverage rather than being restricted to having partial coverage. The specific values of $p_{2}$ at which this 
occurs will, of course, depend on the exact value of the other parameters of the problem. Thus market restrictions may not be the most efficient way of dealing with the problem of catastrophic losses. Whether the combinations of variables that satisfy this condition are of practical importance has yet to be determined.

\section{Discussion}

The assumption that insurers are averse to risk when the scale of variability of underwriting results encompasses events that would lead to bankruptcy is sufficient to establish the need for premiums to reflect contingencies. In the case of constant absolute risk aversion, the premium can be characterized as decomposable into three components: the actuarial value of the losses, an allowance for risk aversion, and an allowance for capacity and contingencies. The last component combines capacity and contingencies in such a way that they cannot be separated; if events are not independent then the premium must reflect both elements but if only independent events are of concern then the premium should reflect neither element. The effect of non-independent event probabilities on the supply of insurance is similar to that, described by Venezian [11], of parameter uncertainty on the capital.

The results derived in this analysis lead to the conclusion that the premiums are independent of the capital of the insurer or of the ratio of premium to capital. This conclusion is similar to those reached by using capital asset pricing models or arbitrage pricing theory. Models using mean-variance approaches, such as that of Venezian [10], on the other hand, lead to the conclusion that premium components are related to the capital of the insurer. In the present scheme, capital would enter the supply equation if the utility of the insurer were not exponential, so that under conditions more general than those treated here the capital becomes relevant. It is of interest that even under the assumption of constant absolute risk aversion of the insurers, capital enters the demand side of the equation if it is admitted that the potential purchaser of insurance is concerned about solvency. This can be seen readily by reformulating Equation (2) under conditions in which insolvency matters. Similar behavior has been reported by Doherty and Tinic [2] for pricing based on capital asset pricing models when the purchaser has an interest in solvency.

If risks are segregated into areas subject to independent catastrophic events, with an event in one area affecting all the policies in that area but not those in other areas, the insurer can achieve an economically satisfactory position by offering a given aggregate coverage at a lower premium by diversifying its books across different areas rather than by concentrating in one or more. Note, however, that the model ignores the effect of any operating economies, such as those of scope, concentration, or scale.

It is important to emphasize that the analysis is based on the concept that the supply curves are set in a competitive market but the existence of a stable competitive market is not established. This is not surprising since the structure examined allows for different risk aversion coefficients so firms can be viewed as having different marginal costs of production. The conditions required to ensure that the market exhibits stable competitive equilibria would provide insight into the sustainability of an unregulated market.

The problems cannot, unfortunately, be resolved easily. In fact even the case of non-discriminating monopolistic markets is difficult to handle. A monopolist discriminating on the basis of the risk aversion coefficient of the potential clients will set the price for each insured 
using Equation (2) and would prefer to sell to people with high risk aversion than to those with low risk aversion. Under such conditions the insurer's utility could be optimized at a unique point. For a non-discriminating insurer the problem would be to set a single price which maximizes the expected utility as described by Equation (8), recognizing that the number of people buying coverage at a given price is limited to those who have a risk aversion coefficient larger than that obtained by solving Equation (2) when the price faced by the purchaser is equal to that asked by the insurer. Though the equation to be solved can be set out explicitly in terms of the distribution of risk aversion coefficients in the population, the uniqueness of the solution has not been established.

Even if market stability is not established, however, the results presented here are of interest. They do represent the relation between price and supply that would hold if a monopolist were faced with imminent entry whenever monopolistic profits are being exacted. As such, they also represent premium levels that would be adopted by a regulator who desires stable, non-discriminating, and reasonably competitive premium rates.

\section{Appendix}

\section{Essential properties of the premium function}

The minimum premium given in Equation (9) of the text can be expressed in terms of the function:

$$
(A-1) \quad f(x)=\frac{1}{x} \ln \left(\left(1-a^{\prime}\right) b_{1}^{x}+a^{\prime} b_{2}^{x}\right)
$$

with $0<a^{\prime}<1$.

This appendix sets out the limits of this function as the argument, $x$, tends to zero and infinity, and establishes the monotonicity of the function.

We define:

$$
M=\max \left(b_{1}, b_{2}\right)
$$

$$
m=\min \left(b_{1}, b_{2}\right)
$$

With these definitions we can write Equation $(A-1)$ as:

$$
f(x)=\ln M+\frac{1}{x} \ln \left[a+(1-a) g^{x}\right]
$$


with $0<a<1$ and $0<g<1$.

From Equation $(A-6)$ it is obvious that:

$$
\lim _{x \rightarrow \infty} f(x)=\ln M .
$$

The limit as $x$ tends to zero is $\ln M$ plus $a$ fraction of indeterminate form zero over zero. The limit of this fraction can be found, by l'Hopital's rule, to be $(1-a) \ln g$. Accordingly:

$$
\begin{aligned}
\lim _{x \rightarrow 0} f(x) & =\ln M+(1-a) \ln (m / M) \\
& =a \ln M+(1-a) \ln m .
\end{aligned}
$$

It is apparent that the function is larger at very large values of $x$ than it is at zero. In order to establish that the function is monotonic for all positive $x$, the first derivative must be proved to be positive at all positive values of $x$. This derivative is given by:

$$
f^{\prime}(x)=\frac{x g^{x}(1-a) \ln g-\left(a+(1-a) g^{x}\right) \ln \left(a+(1-a) g^{x}\right)}{x^{2}\left(a+(1-a) g^{x}\right)}
$$

The denominator of $f^{\prime}(x)$ is clearly positive. Denoting the numerator of Equation $(A-9)$ by $h(x)$ it can be seen that $h(0)$ is zero and after some algebra:

$$
h^{\prime}(x)=(1-a) g^{x} \operatorname{lng} \ln \frac{g^{x}}{a+(1-a) g^{x}} .
$$

Since $0<a<1$ and $0<g<1$ the quantities $g$ and $g^{x} /\left(a+(1-a) g^{x}\right)$ are both less than unity for positive values of $x$; it follows that their logarithms are both negative. The function $h^{\prime}(x)$ is a positive constant times the product of these two logarithms and must, accordingly, be positive for all positive $x$.

The above derivations establish that the function $f(x)$ described by Equation $(A-1)$ is monotonically increasing for positive $x$ and ranges from $\ln M$ to $a \ln M+(1-a) \operatorname{lnm}$.

\section{REFERENCES}

1. COPELAND, T.E., and WESTON, J.F.: Financial Theory and Corporate Policy, Addison-Wesley Publishing Company, Reading, MA, 1979.

2. DOHERTY, N.A., and TINIC, S.M.: "Reinsurance under conditions of capital market equilibrium - A note", Journal of Finance, 34 (September 1981), 949-953.

3. FAIRLEY, W.B.: "Investment income and profit margins in property-liability insurance", Bell Journal of Economics, 10 (Spring 1979), 192-210.

4. FRIEND, I., and BLUME, M.: "The demand for risky assets", American Economic Review, 65 (December 1975), 900-922.

5. HILL, R.D.: "Profit regulation in property-liability insurance", Bell Journal of Economics, 10 (Spring 1979), 172-191. 
6. KENNEY, R.L., and RAIFFA,H.: Decisions with Multiple Objectives:Preferences and ValueTradeoffs, John Wiley and Sons, New York, NY, 1976.

7. KRAUS, A., and ROSS, S.A.: "The determinants of fair profits for the property-liability insurance firm", Journal of Finance, 37 (September 1982), 1015-1028.

8. URRUTIA, J.: "The capital asset pricing model and the determination of fair underwriting returns for the property-liability insurance industry", The Geneva Papers on Risk and Insurance, 11 (January 1986), 44-60.

9. URRUTIA, J.: "An arbitrage pricing theory model for pricing property-liability insurance", paper presented at the Annual Meeting of the American Risk and Insurance Association, Minneapolis, MN, 1984.

10. VENEZIAN, E.C.: "Insurer capital needs under parameter uncertainty", Journal of Risk and Insurance, 50 (March 1983), 19-32.

11. VENEZIAN, E.C.: "Risk, return, and rate regulation in property and liability insurance", Journal of Insurance Regulation, 2 (September 1983), 98-124. 